\title{
Aspectos histopatológicos e imunohistoquímico do sinoviossarcoma em cavidade bucal
}

\author{
Histopathological and immunohistochemical aspects of sinoviosarcoma in oral cavity
}

Aspectos histopatológicos e inmunohistoquímicos del sinovosarcoma en la cavidad

Bárbara Faustino da Silva Teixeira ${ }^{1 *}$; Mirely Duarte Bezerra'; Tayguara Cerqueira Cavalcanti ${ }^{1}$; Igor Lerner Hora Ribeiro²; Camila Maria Beder Ribeiro Girish Panjwani'.

\begin{abstract}
RESUMO
Objetivo: Relatar os aspectos histopatológicos e imunohistoquímicos do sinoviossarcoma em cavidade oral, haja vista que o último relato de cavidade oral foi há mais de 5 anos, este artigo mostra uma atualização sobre essa neoplasia. Detalhamento do caso: Paciente 9 anos de idade do gênero feminino, com sinoviossarcoma localizado em assoalho bucal, assintomático. Foi realizado exames histopatológicos e imunohistoquímicos. Cirurgia de remoção do tumor, e tratamento quimioterápico. Ao estudo imunohistoquímico o caso descrito mostrou positividade para vimentina, citoqueratin Pan e FLI-1. O laudo histopatológico demonstrou se tratar de um sinoviossarcoma do tipo bifásico. Considerações finais: O sinoviossarcoma é uma neoplasia extremamente rara, é um tumor maligno bem definido que representa $5,6 \%$ a $10 \%$ de todos os sarcomas de tecidos moles, o que, portanto, torna seu diagnóstico mais difícil. O histopatológico e a imunohistoquímica auxiliam de forma precisa no diagnóstico, determinando assim, uma boa abordagem terapêutica. O tratamento consiste, habitualmente, na ressecção cirúrgica oncológica; podendo ser seguida de radioterapia, quimioterapia e mesmo à imunoterapia.
\end{abstract}

Palavras-Chave: Sarcoma sinovial, Neoplasias, Sarcoma.

\begin{abstract}
Objective: To report the histopathological and immunohistochemical aspects of oral cavity synoviosarcoma, since the last report of oral cavity was more than 5 years ago. This article shows an update on this neoplasm. Case Detail: A 9-year-old female patient with asymptomatic buccal floor synovinosarcoma. Histopathological and immunohistochemical exams were performed. Tumor removal surgery, and chemotherapy treatment. In the immunohistochemical study the case described showed positivity for vimentin, cytokeratin Pan and FLI-1. The histopathological report was shown to be a biphasic synoviosarcoma. Final Considerations: Synoviosarcoma is an extremely rare neoplasm, a well-defined malignant tumor representing $5.6 \%$ to $10 \%$ of all soft tissue sarcomas, which therefore makes its diagnosis more difficult. Histopathology and immunohistochemistry help precisely in the diagnosis, thus determining a good therapeutic approach. Treatment usually consists of surgical oncologic resection; may be followed by radiotherapy, chemotherapy and even immunotherapy.
\end{abstract}

Key words: Synovial sarcoma, Neoplasms, Sarcoma.

\section{RESUMEN}

Objetivo: informar los aspectos histopatológicos e inmunohistoquímicos del sinoviosarcoma de la cavidad oral, ya que el último informe de la cavidad oral fue hace más de 5 años. Este artículo muestra una

${ }^{1}$ Centro Universitário CESMAC, Maceió-AL. *E-mail: babyfaustinos.t@hotmail.com

${ }^{2}$ Santa Casa de Misericórdia de São Miguel dos Campos-AL.

SUBMETIDO EM: 9/2019

ACEITO EM: 10/2019

PUBLICADO EM: 11/2019

REAS/EJCH | Vol.Sup.36 | e1836 | DOI: https://doi.org/10.25248/reas.e1836.2019 Página 1 de 7 
actualización sobre esta neoplasia. Detalle del caso: paciente de 9 años de edad con sinovinosarcoma de piso vestibular asintomático. Se realizaron exámenes histopatológicos e inmunohistoquímicos. Cirugía de extirpación tumoral y tratamiento de quimioterapia. En el estudio inmunohistoquímico, el caso descrito mostró positividad para vimentina, citoqueratina Pan y FLI-1. El informe histopatológico demostró ser un sinoviosarcoma bifásico. Consideraciones finales: el sinoviosarcoma es una neoplasia extremadamente rara, un tumor maligno bien definido que representa del $5,6 \%$ al $10 \%$ de todos los sarcomas de tejidos blandos, lo que dificulta su diagnóstico. La histopatología y la inmunohistoquímica ayudan precisamente en el diagnóstico, determinando así un buen enfoque terapéutico. El tratamiento generalmente consiste en resección quirúrgica oncológica; puede ser seguido por radioterapia, quimioterapia e incluso inmunoterapia.

Palabras clave: Sarcoma sinovial, Neoplasias, Sarcoma.

\section{INTRODUÇÃO}

O sinoviossarcoma (ss) é um tumor maligno bem definido de tecidos moles que representa $5,6 \%$ a $10 \%$ de todos os sarcomas de tecidos moles. A origem do sarcoma sinovial permanece desconhecida, mas acredita-se que a neoplasia se origine de células mesenquimais pluripotenciais indiferenciadas primitivas, não relacionadas ao tecido sinovial (MEER S, 2003).

O paciente normalmente apresenta uma massa palpável de crescimento lento, que pode crescer durante semanas ou meses, simulando uma lesão benigna (MAHESH KTS, et al., 2013). Podendo apresentar sintomatologia dolorosa ou não.

A SS é mais comum entre os 15 e os 40 anos de idade. Os machos são mais suscetíveis do que as fêmeas com proporções de 1,2: 1. Os locais mais comuns na região de cabeça e pescoço incluem hipofaringe, região pós-faríngea e espaço parafaríngeo. Poucos casos na língua, palato mole, mandíbula, mucosa bucal e assoalho da boca foram descritos na literatura (RAO GV, et al., 2014).

Clinicamente o tamanho do tumor varia de 0,4 a $10 \mathrm{~cm}$ com uma mediana de $4,0 \mathrm{~cm}$ (GAC PE, et al., 2008).

O diagnóstico é estabelecido geralmente baseado na técnica convencional estudo anatomopatológico com coloração de hematoxilina-eosina e suporte complementar com estudo imunohistoquímica, microscopia eletrônica ou biologia molecular (GAC PE, et al., 2008).

O tratamento consiste, habitualmente, na ressecção cirúrgica oncológica; podendo ser seguida de radioterapia, quimioterapia e mesmo à imunoterapia. Os sarcomas sinoviais de cabeça e pescoço devem ser submetidos a ressecção cirúrgica completa seguida de radioterapia pós-operatória naqueles com alto risco de recorrência locorregional. Em termos de quimioterapia, também existem dados emergentes sobre sua eficácia no tratamento do sarcoma sinovial da cabeça e pescoço (MENDONÇA GS et al, 2015; NANCY L e EDWARD S, 2014).

Muitos estudos sugerem que a radioterapia adjuvante tem boa influência nas taxas de recorrência. Alguns autores indicam radioterapia em sarcomas de tecidos moles de alto grau maiores que $5 \mathrm{~cm}$, reduzindo assim as chances de recorrência local (BAPTISTA AM, 2006).

O prognóstico para sarcoma sinovial tem relação com índice mitótico, tamanho do tumor, idade, presença de áreas pouco diferenciadas, de áreas rabdóides e de áreas extensas de necrose. Além disso, alto índice de proliferação celular é considerado fator adverso para mortalidade relacionado à doença (LIMA FO, et al., 2005).

Diante disso, o presente estudo tem por objetivo relatar um caso incomum de sinoviossarcoma em cavidade bucal, visando seus aspectos histopatológicos, imunohistoquímico e radiográficos. Dessa forma, levar a outros profissionais mais conhecimentos acerca do tema, haja vista que o último relato de cavidade oral foi há mais de 5 anos, e a pesquisa mostra uma atualização sobre essa neoplasia. 


\section{RELATO DE CASO}

Paciente leucoderma, 9 anos de idade, gênero feminino, residente da cidade de Maceió-AL. Foi encaminhada para a Santa Casa de Misericórdia de São Miguel dos Campos, na anamnese, não relatou sintomatologia dolorosa, ao exame intra-oral apresentou aumento de volume na região de assoalho bucal, com crescimento lento (Figura 1).

\section{Figura 1- Aspecto clínico do tumor}

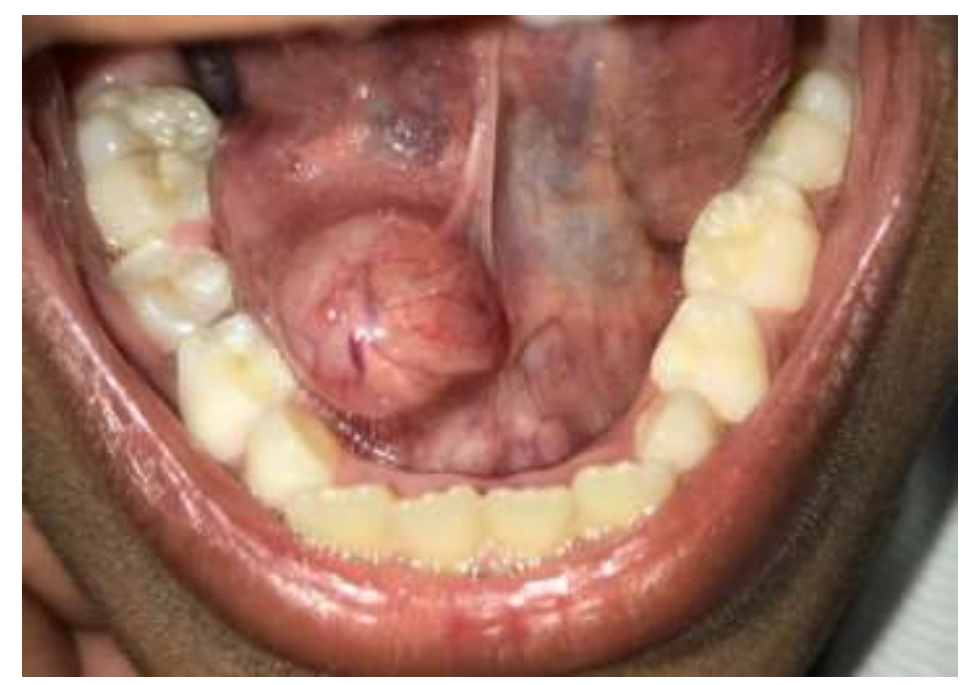

Fonte: Ribeiro ILH, 2017.

Inicialmente a hipótese clínica foi de adenoma pleomórfico. Foi realizada uma excisão cirúrgica, retirando todo o tumor, e da área circundante atingindo cerca de $11,8 \times 2,8 \mathrm{~cm}$ aproximadamente (Figura 2).

Figura 2- fotografia da peça macroscópica mostrando seu comprimento.

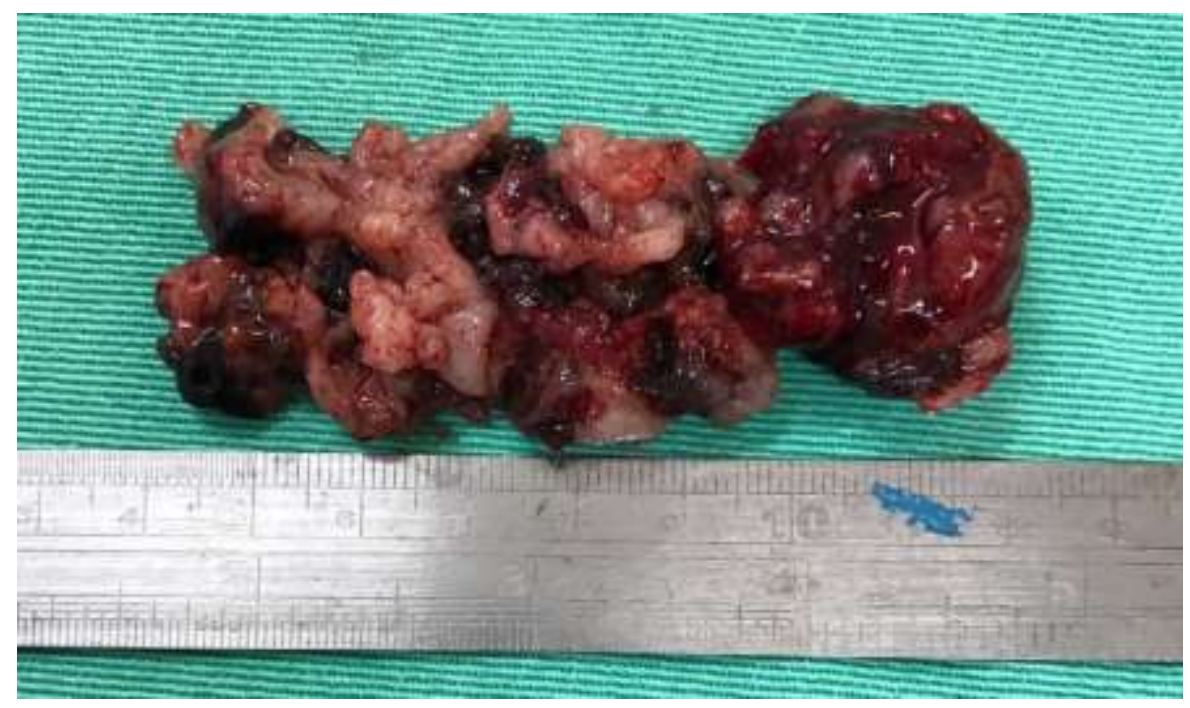

Fonte: Ribeiro ILH, 2017. 
Figura 3- corte histopatológico, corado em hematoxilina e eosina (100x)

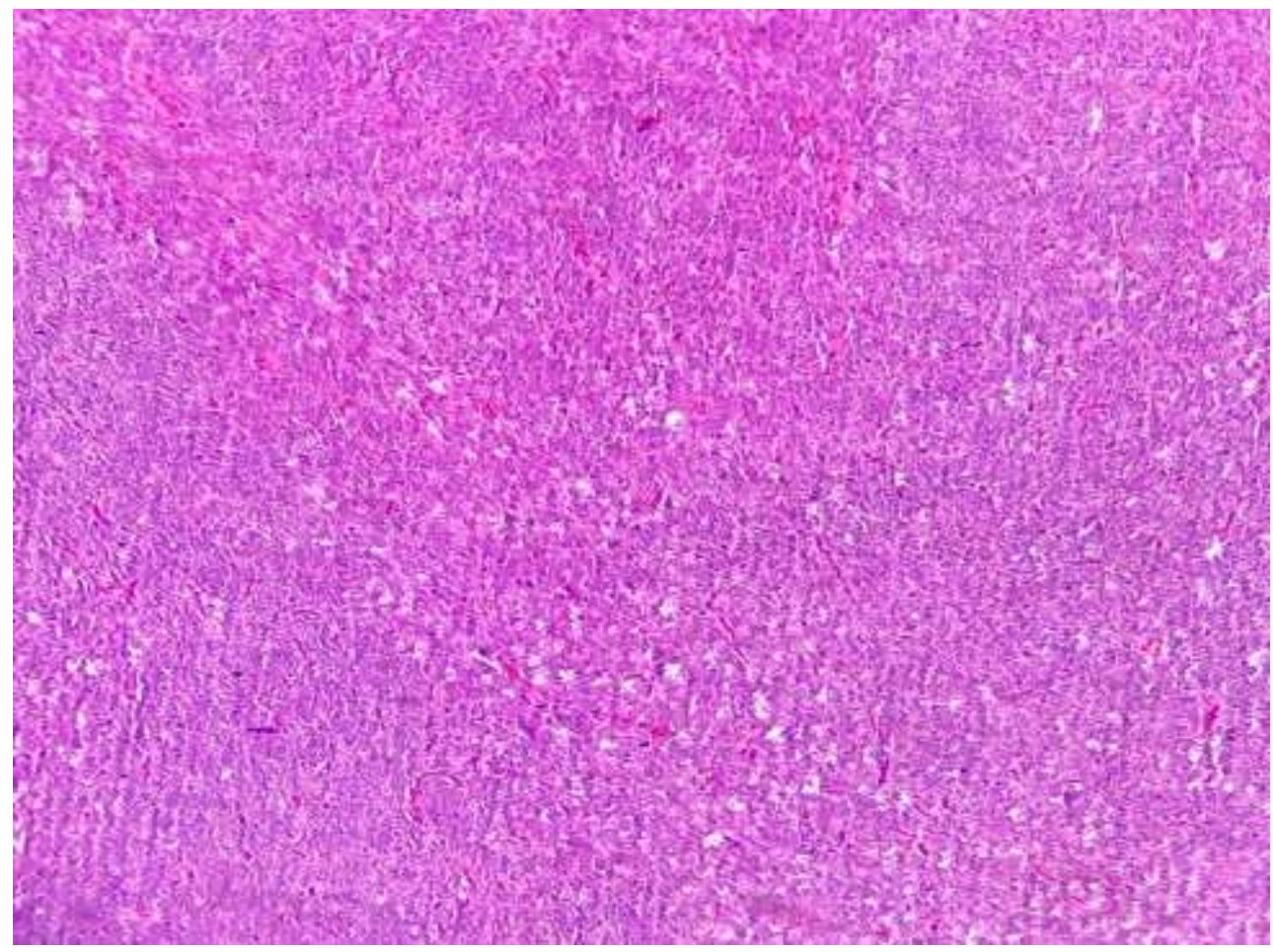

Fonte: Laboratório CEPAMA, 2017.

Figura 4- corte histopatológico, corado em hematoxilia e eosina (400x).
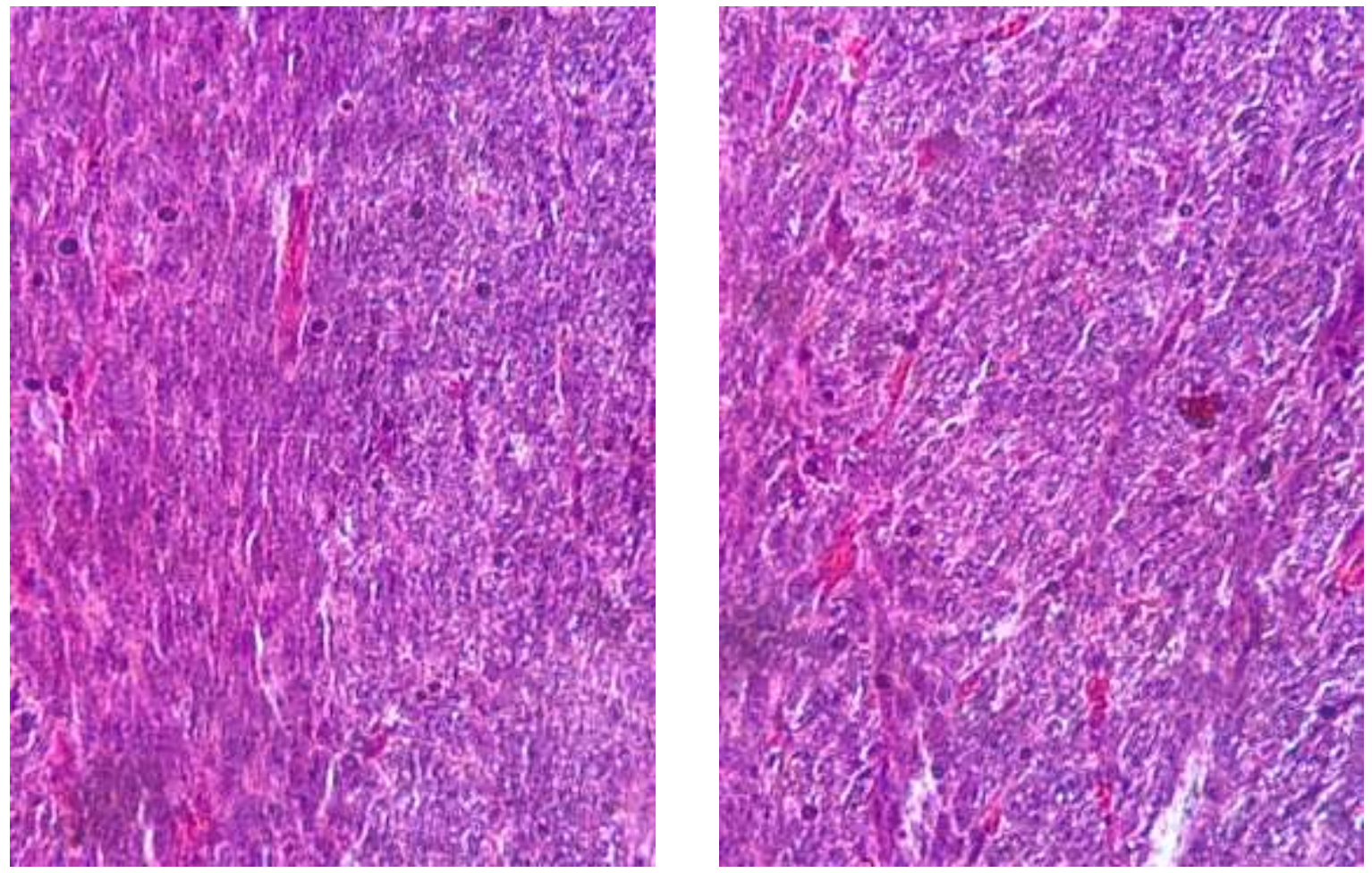

Fonte: Laboratório CEPAMA, 2017. 
A peça foi enviada para estudo histopatológico (Figuras 3 e 4). A análise com cortes corados com hematoxilina e eosina (HE) foi realizada, revelando células fusiformes contendo núcleos atípicos, por vezes pleomórficos e hipercromáticos dispostos em feixes que se cruzam em várias direções, sendo classificado no tipo bifásico.

Para realização de reações Imunohistoquímicas são utilizados métodos automatizado de desparafinização, hidratação e recuperação antigênica em etapa única, com os seguintes anticorpos: vimentina; proteína S-100; citoceratina Pan; miogenina; CD99 (Cluster of differentiation 99) ; FLI-1 (Friend leukemia integration 1) e Desmina (Tabela 1).

Tabela 1- Marcadores tumorais para diagnóstico em imunohístoquimica.

\begin{tabular}{lc} 
Vimentina (clone V9) & Positivo \\
\hline Proteína S-100 (policional Rabbit) & Negativo \\
\hline Citoqueratina Pan (clone AE1/ AE3) & Positivo \\
\hline Miogenina (cloneF5D) & Negativo \\
\hline CD99 (clone 12E7) & Negativo \\
\hline FLI-1 ( clone MRQ-1) & Positivo \\
\hline Desmina (clone D33) & Negativo \\
\hline
\end{tabular}

Fonte: Laboratório CEPAMA, 2017.

Após o laudo histopatológico e imunohistoquímico o tratamento de escolha foi a quimioterapia seguida da cirurgia de ampliação de margens do tumor, e acompanhamento periódico.

Atualmente, a paciente encontra-se ainda em protocolo de quimioterapia, em um período de observação de aproximadamente 2 anos. Porém, com fator prognostico bom.

\section{DISCUSSÃO}

O sarcoma sinovial (ss) é uma neoplasia rara encontrada nos tecidos moles, a literatura descreve uma maior frequência de apresentação no sexo masculino e uma apresentação menos dolorosa na localização intraoral do que nos demais locais (GAC PE, et al., 2008). O que difere do caso discutido, que se trata do gênero feminino, a paciente não relatou dor ou sensibilidade.

Os pacientes são mais freqüentemente afetados nos primeiros anos de vida (BERTOLINI F, et al., 2013). Os dados apresentados corroboram com os dados da literatura, por se tratar de uma paciente jovem. Além disso, a neoplasia evoluiu em um período de 1 ano.

Baseados nos componentes celulares os sinoviossarcomas são subclassificados em três grupos: célula epitelial monofásica, célula fusiforme monofásica e tipo bifásico. Histologicamente o sinoviossarcoma bifásico contém componentes celulares epiteliais e fusiformes distintos, mas interligados. O tipo epitelial monofásico consiste de células colunares cuboidais altas ao redor dos espaços glandulares e dispostas em ninhos sólidos. As células são caracterizadas por núcleos grandes, redondos ou ovais e abundante citoplasma pálido com células distintas. O lúmen glandular geralmente contém mucina epitelial. O monofásico fusiforme consiste de células fusiformes bem orientadas, bastante arredondadas, pequenas e uniformes, com uma alta relação citoplasmática nuclear (RAO GV, et al., 2014).

A vimentina é uma proteína dos filamentos intermediários, é usado como marcador para tecidos derivados do mesoderma e, como tal, pode ser usada como um marcador imunohistoquímico para sarcomas. Além disso, é geralmente expressa no sarcoma de células fusiformes. 
no caso relatado apresentou positividade comprovando se tratar de um sinoviossarcoma (QUEIROZ LS e ZANARDI VA, 2018; GARCÍA PA, et al., 2012).

As células tumorais em geral são focalmente positivas para proteínas S-100. É um anticorpo útil para definição de tecido de origem de uma neoplasia fusocelular (QUEIROZ LS e ZANARDI, VA, 2018; WERNER B, et al., 2005). A proteína S-100 apresentou negatividade no presente caso, visto também na maioria dos casos relatados.

A citoceratina Pan (AE1/AE3) é uma mistura de 2 anticorpos monoclonais, AE1 reconhece a maioria das queratinas ácidas (tipo I), já AE3 reconhece todas queratinas básicas conhecidas (tipo II). Este anticorpo reconhece polipéptidos de citoqueratina de quase todos os epitélios, sendo útil para diferenciar tumores epiteliais (carcinomas) de tumores não epiteliais (linfomas, melanomas ou sarcomas), sendo a sua marcação geralmente negativa para os últimos (QUEIROZ LS, ZANARDI, VA, 2018; LOPES C, CORDEIRO M. 2018). Apresenta positividade para $97 \%$ de todos os sarcomas sinoviais, incluindo neste relato, caracterizando 0 tumor em bifásico.

A miogenina é um marcador de diferenciação muscular esquelética para rabdomiossarcoma. Realizada a imuno-histoquímica da miogenina para descartar outros tumores musculares, como rabdomiossarcomas, que geralmente são positivos para miogenina (enquanto que os sarcomas sinoviais são geralmente negativos para miogenina) (HALDAR F, et al., 2007).

O CD99 é usado para distinguir o sarcoma de Ewing de outros tumores de aparência histológica similar, bem como para a identificação de tumores tímicos e de tumores fusiformes, como sarcoma sinovial, hemangiopericitoma e meningioma. A expressão do marcador CD99 foi relatada em neoplasias de células fusiformes de tecido conjuntivo, como SS (RIBEIRO ILA, et al., 2017). Porém no caso relatado o marcador tumoral CD99 apresentou negatividade.

O fator de transcrição nuclear FLI-1 tem sido proposto como uma ferramenta útil no diagnóstico diferencial de pequenos sarcomas de células redondas. Recentemente tem sido relacionada à presença de padrões no promotor do gene FLI1 a uma resposta anormal celular. Considerando que a literatura experimental tem reportado que variantes no FLI1 podem levar ao desenvolvimento de hipoplasia, é possível sustentar a possibilidade de que determinadas estruturas nesse gene possam influenciar o risco de um indivíduo (SAWITZI FR, 2012).

A desmina é usada como marcador de tumores de musculatura lisa e esquelética (leiomiossarcomas, rabdomiossarcomas) (BACCHI, 2019). São fortemente marcadas para células neoplásicas, porém apresentou negatividade.

Os pacientes com tumores maiores que $5,0 \mathrm{~cm}$ de diâmetro apresentam maior risco de recidiva tumoral local, metástases à distância e mortalidade do que aqueles com tumores menor que $5,0 \mathrm{~cm}$ de diâmetro (WUSHOU A, MIAO X, 2014). Pelo fato de o tumor apresentar um diâmetro maior que $5,0 \mathrm{~cm}$, e envolver uma localização intraoral, mostra que o caso tem um prognóstico pior, e que há chances de haver recidivas.

Wushou A e Miao X (2014) relataram um resultado mais favorável em pacientes com tumores bifásicos, enquanto outros grupos não encontram diferenças na sobrevida entre pacientes com tumores monofásicos e aqueles com tumores bifásicos.

Os dados desse caso não mostram índice mitótico na amostra analisada, portanto se limita falar sobre grau prognóstico nesse paciente relacionado a índice mitótico.

A ressecção cirúrgica ampla é a principal opção de tratamento para sinoviossarcoma. Porque a excisão completa dos tumores intra-orais é nem sempre é possível, uma abordagem terapêutica multimodal consistindo em excisão local radical extensa, pós-operatório radioterapia e quimioterapia é frequentemente recomendada (MAHESH KTS, et al., 2013).

\section{CONSIDERAÇÕES FINAIS}


O sinoviossarcoma é uma neoplasia extremamente rara, o que, portanto, torna seu diagnóstico mais difícil. Os dados deste caso clínico demonstraram que o sinoviossarcoma pode ser um diagnóstico considerado no gênero feminino em idade jovens, como diagnóstico diferencial de outras lesões. O histopatológico e a imunohistoquímica auxiliam de forma precisa no diagnóstico, determinando assim, uma boa abordagem terapêutica.

\section{REFERÊNCIAS}

1. BAPTISTA AM, et al. Synovial sarcoma of the extremities: prognostic factors for 20 nonmetastatic cases and a new histologic grading system with prognostic significance. Clinics, 2006; 61: 381-386.

2. BERTOLINI F, et al. Synovial cell sarcoma of the neck. Case report and review of the literature. Acta Otorhinolaryngol Ital, 2003; 23: 391-395.

3. GAC PE, et al. Sarcoma sinovial intraoral primario monofásico. Revista chilena de cirugía, 2008; 60: 326-331.

4. GARCÍA PA, et al. Sarcoma sinovial bifásico primario de pulmón: reporte de un caso y revisión de la literatura. Cirugía y Cirujanos, 2012; 80: 67-71

5. HALDAR M, et al. Um modelo de mouse condicional de sarcoma sinovial: informações sobre uma origem miogênica. Câncer cell, 2007; 11: 375-388.

6. LIMA FO, et al. Regiões organizadoras nucleolares argirofílicas no sarcoma sinovial. Jornal Brasileiro de Patologia e Medicina Laboratorial, 2005; 41: 347-52.

7. LOPES C, CORDEIRO M. Imunohistoquimica: anticorpos primários, 2018. PATHOLOGIKA. Disponível em: https://pathologika.com/imuno-histoquimica/anticorpos-primarios/citoqueratina-ae1-ae3/. Acesso em: 27 de setembro 2019.

8. MAHESH KTS, et al. Synovial Sarcoma of the Buccal Mucosa: A Rare Case Report. Case reports in dentistry, 2013; p.5.

9. MEER S, et al. Oral synovial sarcoma: A report of 2 cases and a review of the literature. Oral Surgery, Oral Medicine, Oral Pathology, Oral Radiology, and Endodontology, 2003; 96: 306-315.

10. MENDONÇA GS, et al. Monophasic synovial sarcoma- case report. Revista Científica da FMC, 2015; 10:260-264.

11. QUEIROZ, LS; ZANARDI, VA. Imunohistoquímica.2018.in: UNICAMP. 2018. Disponível em: $<$ http://anatpat.unicamp.br/nptppnet5b.html> Acesso em: 01 abril. 2019.

12. RAO GV, et al. Primary biphasic synovial sarcoma of gingiva: Report of a rare case. Journal of oral and maxillofacial pathology, 2014; 18: 1-10.

13. RIBEIRO ILA, et al. Sarcoma sinovial: relato de caso. Revista Gaúcha de Odontologia, 2017; 65: 260-264.

14. SAWITZKI FR. Haplótipos de diferentes SNPs no interior do gene FLI1 em indivíduos afetados e não-afetados pelo sarcoma de Ewing (Mestrado em Biologia Celular e Molecular)- Pontifícia Universidade Católica do Rio Grande do Sul, Porto Alegre, 2012; 39.

15. WERNER B, et al. Uso prático da imuno-histoquímica em patologia cirúrgica, Rio de Janeiro. Jornal Brasileiro de Patologia e Medicina Laboratorial, 2005; 41: 353-364.

16. WUSHOU A, MIAO X. Tumor size predicts prognosis of head and neck synovial cell sarcoma. Oncology Letters, $2015 ; 9(1): 381-386$. 